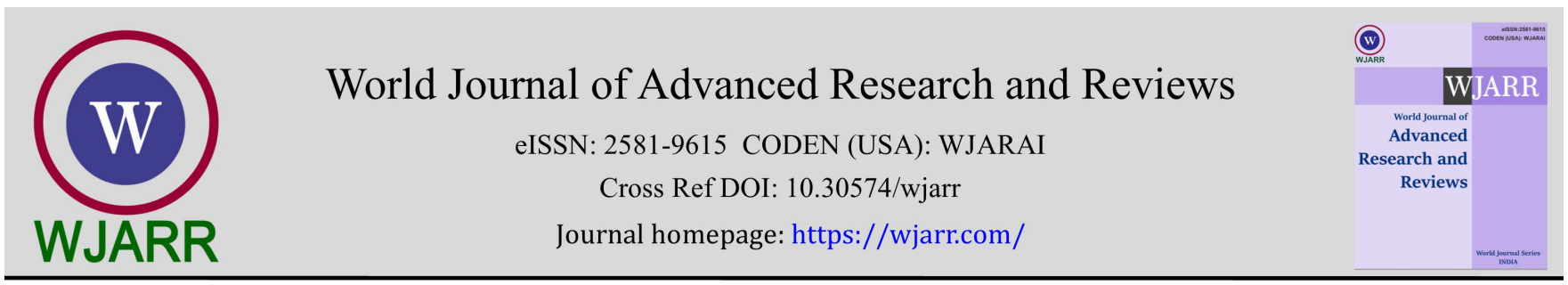

(REview ARTICLE)

Check for updates

\title{
What if Bevacizumab was discontinued! Can IMpower130 be exchanged for IMpower150 for advanced non-squamous NSCLC patients with PD-L1 <50\% and without genomic mutations?
}

\author{
Khaled Elmahdi Omran 1,2,* \\ ${ }^{1}$ Oncology Department, Tobruk Medical Center, Tobruk, Libya. \\ ${ }^{2}$ Faculty of Medicine, University of Tobruk, Tobruk, Libya.
}

World Journal of Advanced Research and Reviews, 2021, 11(02), 291-297

Publication history: Received on 20 July 2021; revised on 23 August 2021; accepted on 25 August 2021

Article DOI: https://doi.org/10.30574/wjarr.2021.11.2.0398

\begin{abstract}
There was a radical change in the first-line management of advanced NSCLC with negative genetic oncological drive in the last 5 years. Immune checkpoint inhibition is currently recommended for such a group of patients by major international guidelines devoted to lung cancer, as long as there is no contraindication. The recommendations came as a single agent of immune checkpoint inhibitor, combination of an immune checkpoint inhibitor with chemotherapy with an optional anti-angiogenic agent, or combination between two different immune checkpoint inhibitors; based on the level of expression of programmed death-ligand 1 in the tumour microenvironment and the type of immune checkpoint inhibitor is intended to be used. IMpower150 was a clinical trial that illustrated the effectiveness of the addition of Atezolizumab (immune checkpoint inhibitor) to chemotherapy and Bevacizumab (anti-angiogenic agent) in treatment naïve advanced non-squamous NSCLC patients with no genetic aberrations. In the same trial, there was no significant difference between chemotherapy plus either Atezolizumab or Bevacizumab. Moreover, Atezolizumab experienced other disappointing results in different clinical trials in NSCLC and other malignancies such as triple-negative breast cancer when combined with chemotherapeutic agents that require corticosteroids as pre-medications during therapy. This review evaluates the synergistic anti-neoplastic effect of immune checkpoint inhibitor and anti-angiogenic agent in NSCLC which presented in IMpower150 by Atezolizumab and Bevacizumab, especially this combination is the preferred option for other malignancies such as hepatocellular carcinoma and renal cell carcinoma. Additionally, the review overlooks the impact of corticosteroids on Atezolizumab in different clinical trials, particularly in NSCLC.
\end{abstract}

Keywords: NSCLC; Immune Checkpoint Inhibitors; Anti-angiogenesis; Atezolizumab; IMpower150; IMpower130

\section{Introduction}

There was a tremendous treatment paradigm shift in advanced NSCLC with no driver mutations throughout the last decade, particularly after the adoption of the immune checkpoint blockade to exploit the host immune response to take over the battle against cancer from chemotherapy. Similar to any debut of treatment intervention, immune checkpoint inhibitors were investigated primarily as a salvage therapy beyond the 1st line of treatment in advanced NSCLC, when they showed superiority over chemotherapy in 2 nd line setting with manageable side effects. That led to the promotion of these agents to treatment-naïve advanced NSCLC patients. Within few years since the engagement of immuneoncology (IO) in lung cancer that were packed with clinical trials, the immune checkpoint inhibitors became the preferred 1st line option for many NSCLC patients who are in the late stages of the disease and whose tumours do not harbour sensitising genomic aberrations [1]. Pembrolizumab, the inhibitor of the programmed death receptor (PD)-1 and Atezolizumab, the inhibitor of the programmed death-ligand (PD-L) 1 were approved as a single agent or in

\footnotetext{
* Corresponding author: Khaled Elmahdi Omran; Email: kelmahdiomran@gmail.com Oncology Department, Tobruk Medical Centre, Tobruk, Libya. 
combination with chemotherapy according to the percentage of PD-L1 expression in the tumour (PD-L1 $\geq 50 \%$ ). Nivolumab, the PD-1 inhibitor was only approved in conjunction with another checkpoint inhibitor (Ipilimumab, the inhibitor of cytotoxic Tcell lymphocyte-associated protein 4 [CTLA-4]) and chemotherapy. More recently, Cemiplimab, which targets PD-1, was approved for 1st line treatment of advanced NSCLC in tumours having PD-L1 expression of $\geq$ $50 \%$ with no aberration in epidermal growth factor receptor (EGFR), anaplastic lymphoma kinase (ALK), and ROS proto-oncogene 1, receptor tyrosine kinase (ROS1) [2].

Atezolizumab was the only immune checkpoint inhibitor to be approved for the 1st line treatment of the same disease combined with chemotherapy and Bevacizumab, the anti-angiogenic agent. The trial of IMpower150 where Atezolizumab and Bevacizumab were added to chemotherapy indicated that concurrent administration of Atezolizumab and Bevacizumab exerted synergistic anti-cancer effects [3].

Atezolizumab is a humanized immunoglobulin 1 (IgG1) monoclonal antibody with specially engineered crystallizable fragment $(\mathrm{Fc})$ domain to abolish antibody-dependent cellular cytotoxicity and preventing depletion of activated T cells and antigen-presenting cells which also express an alternative receptor for PD-L1, the B7.1 receptor [4,5]. Bevacizumab is a humanized anti-vascular endothelial growth factor (VEGF) monoclonal antibody that binds to and neutralizes all human VEGF-A [6]. VEGF-expressing tumours were previously reported to have a worse prognosis than those with negative VEGF expression, including lung cancer [7]. Not only promotes neovascularisation for tumour growth, but VEGF also has its suppressive effect on T cells and has a role in modulating tumour immune-microenvironment to an immunosuppressive state [8]. It's worth mentioning that, in advanced NSCLC, anti-VEGF therapy increases the risk of pulmonary haemorrhage which could be fatal, squamous cell histology in particular [9].

The potential immunosuppressive properties of corticosteroids led to an acceptable concern about the administration of such medications during therapy which might influence the efficacy of ICIs. Therefore, it was uniformly an exclusion criterion in clinical trials if the candidate was on a certain level of corticosteroids therapy before the enrolment. However, the impact of co-administration of corticosteroids on ICIs was always a controversial subject. [9-13]. This review is appraising the option of combining ICI with anti-VEGF for treatment-naïve advanced non-squamous NSCLC patients whose tumours have no genomic mutations. Moreover, the discussion will highlight the influence of corticosteroids on Atezolizumab in the context of other clinical trials combining Atezolizumab with chemotherapy such as IMpower130 and IMpower132, especially if anti-VEGF therapy is contraindicated.

\section{IMpower150}

Undoubtedly, IMpower150 was an iconic immunotherapy trial in the effort of fighting advanced NSCLC by adding Atezolizumab and Bevacizumab together with chemotherapy to the field of the tumour microenvironment. To our knowledge, it was the first time for an immune checkpoint inhibitor to be combined with an anti-angiogenesis drug in a phase III clinical trial dealing with advanced NSCLC. The aforementioned phase III clinical trial enrolled advanced nonsquamous NSCLC patients with/without genetic aberration in EGFR or ALK who were treatment-naïve (patients with EGFR/ALK-positive had disease progression on tyrosine kinase inhibitors before admission). In the trial, patients were randomly assigned in a 1:1:1 ratio to receive atezolizumab plus carboplatin plus paclitaxel (ACP = arm $\mathrm{A}$ of the trial), bevacizumab plus carboplatin plus paclitaxel (BCP = arm $\mathrm{C}$ ), or atezolizumab plus BCP (ABCP = arm B) every 3 weeks for four or six cycles, and atezolizumab, bevacizumab, or both were the maintenance therapy according to their existence on each arm. Stratification was according to sex, presence or absence of liver metastases before enrolment, and PD-L1 tumour expression. On one hand, the benefit of addition of Atezolizumab to the BCP was observed in terms of progression-free survival (PFS), and overall survival (OS) in the intention-to-treat-population, including those with EGFR or ALK genetic mutations and patients with baseline liver metastases, (the median PFS, 8.3 months, arm B vs. 6.8 months, arm C; hazard ratio [HR] for progression of the disease or death, $0.62 ; 95 \%$ confidence interval [CI], 0.52 to 0.74; $\mathrm{P}<0.001$ ); (Median OS, 19.2 months vs. 14.7 months; HR, 0.78; 95\% CI, 0.64 to 0.96; $\mathrm{P}=0.02$, respectively) $[3,14,15]$. On the other hand, the ACP arm of the trial didn't show statistically significant OS when compared with BCP, (HR was 0.88 [95\% CI: 0.72, 1.08; $P=0.204]$ ). Although the trial was designed to investigate the benefit of adding Atezolezumab to BCP to improve the survival of patients with advanced NSCLC, an exploratory third arm was added to the trial (ACP) with the same ratio. Consequently, the trial offered two platforms for comparative statistical investigation (ABCP vs BCP, and ACP vs BCP). Therefore, each platform of the study could be analysed independently.

To begin with, the disclosed data of the addition of Atezolizumab to BCP showed that, PD-L1 inhibitor added more value to the well-known and widely implemented regimen "BCP" in managing patients with advanced non-squamous NSCLC, by increasing the period of disease control and decrease the risk of death from the disease. This regimen (BCP) was implemented after Sandler and colleagues showed in 2006 how adding Bevacizumab to chemotherapy improved PFS and OS of selected patients of NSCLC in 1st line setting, although with an increased risk of fatal pulmonary haemorrhage 
[9]. The ingenious notion of adding an immune checkpoint inhibitor to an anti-angiogenic agent plus chemotherapy might have a synergic anti-neoplastic effect was proven to be factual by IMpower150.

Additionally, vigorous information was extrapolated from the trial about patients with liver metastases. The combination of Atezolizumab with BCP had a trend of improving the survival of patients with liver metastases compared to the control arm of BCP [3,14]. It is noteworthy that, subgroup analysis of the trial by Sandler, et al. investigated chemotherapy with or without Bevacizumab illustrated the beneficial addition of Bevacizumab to the patients with liver metastases [9]. Subsequently, the addition of Atezolizumab to BCP augmented the role of Bevacizumab with extra extension in survival for advanced no-squamous NSCLC patients with liver metastases. Interestingly, the permission to include patients with EGFR/ALK genomic mutation in the trial was a venturesome decision; particularly, when EGFR/ALK aberrations are considered a negative prognostic marker of response to the ICIs [16]. Yet again, what was derived from the data of those patients was unanticipated as there was a trend of improvement in their survival when Atezolizumab was added to BCP. Consequently, these results would give EGFR/ALK-positive none-squamous NSCLC patients a salvage option after their tumours got resistant to tyrosine kinase inhibitors (TKIs) [3,15].

Nonetheless, the undermined part of the trial which compared between ACP arm and BCP arm has its significant implication. It is not only because of the failure of Atezolizumab to add any survival benefit, however, but it also extends to the oncological clinical practice, when Bevacizumab is contraindicated or adverse events of anti-VEGF emerge during the course of therapy if $\mathrm{ABCP}$ was chosen for 1st line management. Therefore, anticipating adverse effects of anti-VEGF therapy such as hypertension, or excessive haemoptysis that might lead to pulmonary haemorrhage is crucial in selecting the right patient for ABCP. Discontinuing Bevacizumab during therapy with ABCP due to complications of Bevacizumab would leave the patient on the statistically inefficient regimen of ACP compared to ABCP or BCP. To shed a light on what could be suggested in such situations, reviewing the chemotherapy backbones used in the clinical trials that combined IO with chemotherapy and evaluating the diversity between them is essential.

\section{The paramount regimen of chemotherapy before the introduction of ICI in NSCLC}

Before the introduction of immune-oncology in the management of lung cancer, multiple lines of platinum-based chemotherapy regimens were investigated against each other for the 1st line therapy of advanced NSCLC to select the paramount protocol based on PFS and OS with manageable toxicities. In 2006, Ohe, et al. presented their results of the phase III trial where they investigated four different chemotherapeutic agents combined with a platinum agent for 1st line treatment in advanced NSCLC patients (cisplatin plus irinotecan versus carboplatin plus paclitaxel, cisplatin plus gemcitabine, and cisplatin plus vinorelbine). Their final analysis showed that all four regimens have similar efficacy [17]. Well along, nanoparticle-albumin bound (nab)-paclitaxel combined with carboplatin was found to be as effective as solvent-based paclitaxel plus carboplatin in NSCLC by a phase III trial [18,19]. It was established that platinum-based doublet regimen is the best treatment for advanced NSCLC regardless of what agent would be combined with platinum, until the admission of the concept of maintenance therapy after the induction phase of treatment. The key change came after the addition of Bevacizumab to chemotherapy and continued as maintenance therapy showed significant survival improvement to platinum-based doublet chemotherapy [9]. Likewise, as pemetrexed was introduced to non-squamous NSCLC, it showed significant survival advantage, with less haematological adverse events, as compared with other platinum-based regimens [20,21]; the survival of such patients was augmented further by keeping pemetrexed for maintenance therapy after it was combined in induction phase with platinum agent [22]. Furthermore, switch maintenance to pemetrexed after induction period using a platinum plus chemotherapeutic agent other than pemetrexed was approved in advanced non-squamous NSCLC [Ciuleanu]. Additionally, the PRONOUNCE trial compared the efficacy of pemetrexed plus carboplatin followed by maintenance pemetrexed with paclitaxel plus carboplatin plus bevacizumab followed by maintenance bevacizumab in patients with advanced non-squamous NSCLC and concluded there was no significant difference between both regimens concerning PFS and OS [23]. As a result, these trials welldefined the paramount 1st line management of advanced NSCLC before the approval of ICI in the 1st line setting, and that was either carboplatin plus paclitaxel with Bevacizumab for induction and maintenance, or platinum plus pemetrexed or any other approved chemotherapeutic agent followed by maintenance pemetrexed.

\section{IMpower130}

The chemotherapy regimen used concurrently with Atezolizumab in IMpower130 was not an optimal choice. Although this chemotherapy regimen was similar for the control arm (carboplatin + nab-paclitaxel), switch maintenance therapy with pemetrexed was permitted in the control arm only. Nonetheless, the addition of Atezolizumab to chemotherapy followed by Atezolizumab as maintenance therapy without pemetrexed improved OS and PFS significantly (OS HR 0.79 [95\% CI 0.64-0.98], p= 0.033; PFS HR 0.64 [95\% CI 0.54-0.77], $\mathrm{p}<0.0001$ ). The number of patients who were allowed 
to receive maintenance pemetrexed out of 232 received the assigned therapy in the control arm, was not mentioned in the trial summary. The information disclosed about pemetrexed therapy in the trial was only about the mean duration of therapy and the mean number of cycles of pemetrexed received (mean duration $=4.9$ months, [standard deviation $(S D)=4.1]$; mean number of doses = 7.7, [SD = 5.6]) [24]. On one hand, the addition of pemetrexed to Atezolizumab during the maintenance period of the investigational arm of the IMpower130 trial would have augmented the PFS and OS benefit further, theoretically. This strategy was actually implemented in KEYNOTE-189. In KEYNOTE-189, the assumption was that adding Pembrolizumab to platinum plus pemetrexed followed by maintenance Pembrolizumab and pemetrexed would make improvement in PFS and OS to chemotherapy alone, and indeed, it did. The median OS of Pembrolizumab plus chemotherapy arm was 22.0 (19.5 to 25.2) months, and for placebo plus chemotherapy was 10.7 (8.7 to 13.6) months with HR, 0.56; (95\% CI, 0.45 to 0.70); and Median PFS was 9.0 (8.1 to 9.9) months and 4.9 (4.7 to 5.5) months, respectively (HR, 0.48; [95\% CI, 0.40 to 0.58]) [25,26]. The clinical interpretation of that was Pembrolizumab decreased the risk of disease progression by $52 \%$ (16\% more than IMpower130), and risk of death by $44 \%$ (23\% more than IMpower130), when it was added to chemotherapy for 1st line management of advanced nonsquamous NSCLC. The advantage gained by KEYNOTE-189 over IMpower130 could be theoretically attributed to the concurrence addition of pemetrexed to pembrolizumab during the maintenance phase in KEYNOTE-189. On the other hand, one of the well-known adverse effects of pemetrexed is the skin toxicity manifested as skin rash and pruritis $[27,28]$. To mitigate the toxicity of pemetrexed, it was advised by the manufacturer of pemetrexed to administer dexamethasone $4 \mathrm{mg}$ twice daily for 3 days starting the day before each pemetrexed administration [29]. Consequently, frequent administration of corticosteroids during the maintenance period of therapy would be inevitable. It is still controversial whether corticosteroids impede the mechanism of action of immune checkpoint inhibitors [30]. Although co-administration of corticosteroids with Pembrolizumab didn't hinder its immune checkpoint inhibition, there were several incidents when Atezolizumab showed negative results in clinical trials when the design of the trial mandated the administration of corticosteroids as a pre-treatment. For instance, IMpower132 had a similar treatment protocol of KEYNOTE-189 apart from the substitution of Atezolizumab for Pembrolizumab. Atezolizumab significantly enhanced PFS when it was added to platinum plus pemetrexed and co-administered with pemetrexed in the maintenance phase; however, it couldn't sustain that advantage for OS [31]. If pemetrexed was not set to be administered during the maintenance period of IMpower132 (subsequently there would be no administration of corticosteroids), would be there a significant improvement in OS, similar to what was observed in IMpower130 when there was no switch maintenance of pemetrexed administered with Atezolizumab? Another example of the negative impact of corticosteroids on Atezolizumab could be derived from IMpassion130 and IMpassion131. In these two trials, Atezolizumab was combined with either nab-paclitaxel (IMpassion130), or paclitaxel (IMpassion131) in advanced triple-negative breast cancer, and the investigation was to detect if the addition of Atezolizumab would improve in PFS and OS to the single taxane agent. The addition of Atezolizumab to nab-paclitaxel significantly improved PFS with a clinically meaningful improvement in OS was noticed in the PD-L1-high subgroup in comparison to nab-paclitaxel alone [32]. When Atezolizumab was added to paclitaxel, it did not show any difference in PFS or OS from single-agent paclitaxel, and here the requirement of corticosteroids as premedication for paclitaxel should not be disregarded [33].

Furthermore, the latent discrepancies between PD-1 and PD-L1 inhibitors in terms of efficacy and safety were perceived through the increasing number of trials studying such therapy in NSCLC. Therefore, the cumulative data that is extracted from these trials about ICIs would influence the selection of the drug for clinical practice; particularly, when head to head comparison between ICIs in a clinical trial is not foreseeable. There were two meta-analysis studies compared between PD-1 and PD-L1 inhibitors in NSCLC in 2nd line setting, and they concluded that anti-PD-1 drugs had better performance than anti-PD-L1 ICIs [34,35]. Another meta-analysis by Brito and colleagues based on 13 clinical trials involved ICIs for treatment-naïve advanced NSCLC patients, suggested the superiority of anti-PD-1 over PD-L1 inhibitors when there was a combination with chemotherapy, but as monotherapy, there was no difference between them [36]. Brito hypothetically proposed that the difference could be due to the inhibition of PD-1 which would block both PD-L1 and PD-L2 and that was not achievable by PD-L1 blockade. PD-L2 was described by Latchman et al. to have the ability to inhibit the activation of T-cells [Latchman]. In addition, another point could be extrapolated from the study by Brito and colleagues, which is the presence of chemotherapy in conjunction with PD-1 inhibitors somehow attenuated their efficacy; consequently could be attributed to the co-administration of corticosteroids. All these comparative data has to be taken with caution due to the indirect nature of appraisal and the lack of a trial that compares between ICIs directly. In fact, the design of clinical trials might seem similar or even identical sometimes; nonetheless, the heterogeneity between them makes each one of them a unique experiment. Truthfully, that is applied to IMpower130, IMpower132, IMpower150 and KEYNOTE-189 trials in respect to populations, chemotherapy used in the induction phase, maintenance therapy, and as well as the unidentified variables in each trial of them.

In summary, Impower150 paved the way for researchers to explore the combination of ICIs with anti-angiogenesis therapy further, after the successful synergism of Atezolizumab with Bevacizumab was achieved in advanced nonsquamous NSCLC. Currently, ongoing trials are evaluating other ICIs with different anti-angiogenic agents in advanced 
NSCLC, for instance, Pembrolizumab combination with Ramucirumab (NCT02443324), Pembrolizumab with lenvatinib (NCT03829332), and nivolumab with Bevacizumab (NCT01454102). Another point should the investigators of the IMpower150 be appraised for is the decision to enrol a subgroup of patients with EGFR/ALK-positive NSCLC, who ought to be excluded primarily due to the presumption of being not responders to ICIs. IMpower150 demonstrated that they might benefit from ICIs after the failure of 1st line TKIs, and again that inspired others to recruit such a group of patients in forthcoming trials of ICIs in advanced NSCLC. A phase II trial combining Pembrolizumab with Ramucirumab in EGFR mutant recurrent or metastatic NSCLC is underway (NCT04120454).

Before the time of managing the advanced NSCLC by immune-oncology and after decades of clinical trials, the international societies of oncology defined the platinum-based chemotherapeutic regimen followed by maintenance therapy with either pemetrexed or Bevacizumab if the latter was used in the induction phase, as the standard of care for the advanced non-squamous NSCLC. KEYNOTE-189, IMpower150, and IMpower132 clinical trials investigated this standard of care for advanced NSCLC with or without ICIs. Investigators of the IMpower130 decided not to give pemetrexed during the maintenance period in conjunction with Atezolizumab in the investigational arm of the trial, although that was permitted for the control arm. A hypothetical explanation for not adding pemetrexed to Atezolizumab during the maintenance therapy in the IMpower130 trial could be due to the frequent and scheduled requirement of corticosteroids for pemetrexed, which could have an impact on the efficacy of Atezolizumab. However, the influence of corticosteroids on ICIs, particularly in an intermittent manner is a controversial subject.

The quadruple chemo-immunotherapy regimen that was proposed by IMpower150 (Atezolizumab plus Bevacizumab plus carboplatin plus paclitaxel) is a great choice for advanced non-squamous NSCLC patients, especially those with liver metastases. Regrettably, this is not recommended for patients with a high risk of developing pulmonary haemorrhage or with uncontrolled hypertension. Patients, whose symptoms are controlled, are still eligible for this protocol of therapy as long as they are under close monitoring by the physician. In the event of the emergence of adverse events of an anti-angiogenic agent, stopping Bevacizumab could hinder the patient's survival benefit that could be obtained from ICI by leaving the patient on a statistically ineffectual regimen (ACP). If the probability of the adverse effects of Bevacizumab is limited before the beginning of therapy, nab-paclitaxel could be substituted for paclitaxel and the patient may start Atezolizumab with Bevacizumab plus carboplatin plus nab-paclitaxel. That would leave the patient on another effective regimen (Atezolizumab + carboplatin + nab-paclitaxel), should Bevacizumab has to be omitted during therapy.

\section{Conclusion}

Indeed, IMpower150 enriched the management of advanced non-squamous NSCLC with no genomic aberrations by the quadruple regimen of Atezoliaumab, Bevacizumab, carboplatin and paclitaxel. However, stopping Bevacizumab during the course of therapy due to complications of the anti-angiogenic agent should not impede the survival benefit expected for the patient by leaving the patient on a statistically ineffective regimen of Atezolizumab, carboplatin, and paclitaxel. Earlier trials provided that, there was no difference between paclitaxel and nab-paclitaxel in terms of efficacy. In addition, nab-paclitaxel doesn't require corticosteroids as pre-medication, which controversially could have an impact on Atezolizumab efficacy.

\section{References}

[1] Li Y, Wu YL. The second wave of checkpoint inhibitors with chemotherapy for advanced non-small-cell lung cancer. The Lancet Oncology. 1 Jul 2019; 20(7): 889-91.

[2] National Comprehensive Cancer Network. NCCN clinical practice guidelines in oncology: Non-Small Cell Lung Cancer, Version 4.2021- 3 March 2021.

[3] Socinski MA, Jotte RM, Cappuzzo F, Orlandi F, Stroyakovskiy D, Nogami N, Rodríguez-Abreu D, Moro-Sibilot D, Thomas CA, Barlesi F, Finley G. Atezolizumab for first-line treatment of metastatic nonsquamous NSCLC. New England Journal of Medicine. 14 Jun 2018; 378(24): 2288-301.

[4] Herbst RS, Soria JC, Kowanetz M, Fine GD, Hamid O, Gordon MS, Sosman JA, McDermott DF, Powderly JD, Gettinger SN, Kohrt HE. Predictive correlates of response to the anti-PD-L1 antibody MPDL3280A in cancer patients. Nature. Nov 2014; 515(7528): 563-7.

[5] Vansteenkiste J, Wauters E, Park K, Rittmeyer A, Sandler A, Spira A. Prospects and progress of atezolizumab in non-small cell lung cancer. Expert opinion on biological therapy. 3 Jun 2017; 17(6): 781-9. 
[6] Ferrara N, Hillan KJ, Gerber HP, Novotny W. Discovery and development of bevacizumab, an anti-VEGF antibody for treating cancer. Nature reviews Drug discovery. May 2004; 3(5): 391-400.

[7] Volm M, Koomägi R, Mattern J. Prognostic value of vascular endothelial growth factor and its receptor Flt-1 in squamous cell lung cancer. International journal of cancer. 20 Feb 1997; 74(1): 64-8.

[8] Passiglia F, Reale ML, Cetoretta V, Novello S. Immune-Checkpoint Inhibitors Combinations in Metastatic NSCLC: New Options on the Horizon?. ImmunoTargets and Therapy. 2021; $10: 9$.

[9] Sandler A, Gray R, Perry MC, Brahmer J, Schiller JH, Dowlati A, Lilenbaum R, Johnson DH. Paclitaxel-carboplatin alone or with bevacizumab for non-small-cell lung cancer. New England Journal of Medicine. 14 Dec 2006; 355(24): 2542-50.

[10] Ferrara R, Lai WV, Hendriks L, Sabari JK, Caramella C, Plodkowski AJ, Halpenny D, Chaft JE, Planchard D, Riely GJ. Impact of baseline steroids on efficacy of programmed cell death-1 and programmed death-ligand 1 blockade in patients with non-small-cell lung cancer. J Clin Oncol. 2018; 36: 2872-8.

[11] Fucà G, Galli G, Poggi M, Russo GL, Proto C, Imbimbo M, Ferrara R, Zilembo N, Ganzinelli M, Sica A, Torri V. Modulation of peripheral blood immune cells by early use of steroids and its association with clinical outcomes in patients with metastatic non-small cell lung cancer treated with immune checkpoint inhibitors. ESMO open. 1 Jan 2019; 4(1): e000457.

[12] Petrelli F, Signorelli D, Ghidini M, Ghidini A, Pizzutilo EG, Ruggieri L, Cabiddu M, Borgonovo K, Dognini G, Brighenti M, De Toma A. Association of steroids use with survival in patients treated with immune checkpoint inhibitors: a systematic review and meta-analysis. Cancers. Mar 2020; 12(3): 546.

[13] Aldea M, Orillard E, Mansi L, Marabelle A, Scotte F, Lambotte O, Michot JM. How to manage patients with corticosteroids in oncology in the era of immunotherapy?. European Journal of Cancer. 1 Dec 2020; 141: 239-51.

[14] Reck M, Jotte R, Cappuzzo F, Mok T, West H, Nishio M, Papadimitrakopoulou VA, Orlandi F, Stroyakovskii D, Thomas C, Nogami N. IMpower150: analysis of efficacy in patients (pts) with liver metastases (mets). Pneumologie. Mar 2020; 74(S 01): P44.

[15] Reck M, Mok T, Socinski MA, Jotte RM, Lim DT, Cappuzzo F, Orlandi FJ, Stroyakovskiy D, Nogami N, RodriguezAbreu D, Moro-Sibilot D. 1293P IMpower150: Updated efficacy analysis in patients with EGFR mutations. Annals of Oncology. 1 Sep 2020; 31: S837-8.

[16] Bylicki O, Paleiron N, Margery J, Guisier F, Vergnenegre A, Robinet G, Auliac JB, Gervais R, Chouaid C. Targeting the PD-1/PD-L1 immune checkpoint in EGFR-mutated or ALK-translocated non-small-cell lung cancer. Targeted oncology. Oct 2017; 12(5): 563-9.

[17] Ohe Y, Ohashi Y, Kubota K, Tamura T, Nakagawa K, Negoro S, Nishiwaki Y, Saijo N, Ariyoshi Y, Fukuoka M. Randomized phase III study of cisplatin plus irinotecan versus carboplatin plus paclitaxel, cisplatin plus gemcitabine, and cisplatin plus vinorelbine for advanced non-small-cell lung cancer: Four-Arm Cooperative Study in Japan. Annals of oncology. 1 Feb 2007; 18(2): 317-23.

[18] Socinski MA, Bondarenko I, Karaseva NA, Makhson AM, Vynnychenko I, Okamoto I, Hon JK, Hirsh V, Bhar P, Zhang $\mathrm{H}$, Iglesias JL. Weekly nab-paclitaxel in combination with carboplatin versus solvent-based paclitaxel plus carboplatin as first-line therapy in patients with advanced non-small-cell lung cancer: final results of a phase III trial. J Clin Oncol. 10 Jun 2012; 30(17): 2055-62.

[19] Gupta N, Hatoum H, Dy GK. First line treatment of advanced non-small-cell lung cancer-specific focus on albumin bound paclitaxel. International journal of nanomedicine. 2014; 9: 209.

[20] Grønberg BH, Bremnes RM, Fløtten $\emptyset$, Amundsen T, Brunsvig PF, Hjelde HH, Kaasa S, von Plessen C, Stornes F, Tollåli T, Wammer F. Phase III study by the Norwegian lung cancer study group: pemetrexed plus carboplatin compared with gemcitabine plus carboplatin as first-line chemotherapy in advanced Non-small-cell lung cancer. Journal of clinical oncology. 1 Jul 2009; 27(19): 3217-24.

[21] Li M, Zhang Q, Fu P, Li P, Peng A, Zhang G, Song X, Tan M, Li X, Liu Y, Wu Y. Pemetrexed plus platinum as the firstline treatment option for advanced non-small cell lung cancer: a meta-analysis of randomized controlled trials. PLoS One. 17 May 2012; 7(5): e37229.

[22] Paz-Ares LG, de Marinis F, Dediu M, Thomas M, Pujol JL, Bidoli P, Molinier O, Sahoo TP, Laack E, Reck M, Corral J. PARAMOUNT: Final overall survival results of the phase III study of maintenance pemetrexed versus placebo immediately after induction treatment with pemetrexed plus cisplatin for advanced nonsquamous non-smallcell lung cancer. J Clin Oncol. 10 Aug 2013; 31(23): 2895-902. 
[23] Zinner RG, Obasaju CK, Spigel DR, Weaver RW, Beck JT, Waterhouse DM, Modiano MR, Hrinczenko B, Nikolinakos PG, Liu J, Koustenis AG. PRONOUNCE: randomized, open-label, phase III study of first-line pemetrexed+ carboplatin followed by maintenance pemetrexed versus paclitaxel+ carboplatin+ bevacizumab followed by maintenance bevacizumab in patients with advanced nonsquamous non-small-cell lung cancer. Journal of Thoracic Oncology. 1 Jan 2015; 10(1): 134-42.

[24] West H, McCleod M, Hussein M, Morabito A, Rittmeyer A, Conter HJ, Kopp HG, Daniel D, McCune S, Mekhail T, Zer A. Atezolizumab in combination with carboplatin plus nab-paclitaxel chemotherapy compared with chemotherapy alone as first-line treatment for metastatic non-squamous non-small-cell lung cancer (IMpower130): a multicentre, randomised, open-label, phase 3 trial. The Lancet Oncology. 1 Jul 2019; 20(7): 92437.

[25] Gadgeel S, Rodríguez-Abreu D, Speranza G, Esteban E, Felip E, Dómine M, Hui R, Hochmair MJ, Clingan P, Powell SF, Cheng SS. Updated analysis from KEYNOTE-189: pembrolizumab or placebo plus pemetrexed and platinum for previously untreated metastatic nonsquamous non-small-cell lung cancer. 2020: 1505-1517.

[26] Gandhi L, Rodríguez-Abreu D, Gadgeel S, Esteban E, Felip E, De Angelis F, Domine M, Clingan P, Hochmair MJ, Powell SF, Cheng SY. Pembrolizumab plus chemotherapy in metastatic non-small-cell lung cancer. New England journal of medicine. 31 May 2018; 378(22): 2078-92.

[27] Rusthoven JJ, Eisenhauer E, Butts C, Gregg R, Dancey J, Fisher B, Iglesias J. Multitargeted antifolate LY231514 as first-line chemotherapy for patients with advanced non-small-cell lung cancer: a phase II study. Journal of clinical oncology. Apr 1999; 17(4): 1194.

[28] Villela LR, Stanford BL, Shah SR. Pemetrexed, a novel antifolate therapeutic alternative for cancer chemotherapy. Pharmacotherapy: The Journal of Human Pharmacology and Drug Therapy. May 2006; 26(5): 641-54.

[29] Eli Lilly. Alimta (pemetrexed) package insert. Indianapolis, IN;1/2019.

[30] Sukumar J, Gast K, Quiroga D, Lustberg M, Williams N. Triple-negative breast cancer: promising prognostic biomarkers currently in development. Expert Review of Anticancer Therapy. 2 Jan 2021.

[31] Nishio M, Barlesi F, Ball S, Bordoni R, Cobo M, Dubray-Longeras P, Goldschmidt J, Novello S, Orlandi FJ, Sanborn RE, Szalai Z. 3750 Final efficacy results from IMpower132: First-line atezolizumab+ chemotherapy in patients with stage IV non-squamous NSCLC. Annals of Oncology. 2020.

[32] Schmid P, Adams S, Rugo HS, Schneeweiss A, Barrios CH, Iwata H, Diéras V, Hegg R, Im SA, Shaw Wright G, Henschel V. Atezolizumab and nab-paclitaxel in advanced triple-negative breast cancer. New England Journal of Medicine. 29 Nov 2018; 379(22): 2108-21.

[33] Miles DW, Gligorov J, Andre F, Cameron D, Schneeweiss A, Barrios CH, Xu B, Wardley AM, Kaen D, Andrade L, Semiglazov V. Primary results from IMpassion131, a double-blind placebo-controlled randomised phase III trial of first-line paclitaxel (PAC)+/-atezolizumab (atezo) for unresectable locally advanced/metastatic triplenegative breast cancer (mTNBC).

[34] You W, Liu M, Miao JD, Liao YQ, Song YB, Cai DK, Gao Y, Peng H. A network meta-analysis comparing the efficacy and safety of anti-PD-1 with anti-PD-L1 in non-small cell lung cancer. Journal of Cancer. 2018; 9(7): 1200.

[35] Tartarone A, Roviello G, Lerose R, Roudi R, Aieta M, Zoppoli P. Anti-PD-1 versus anti-PD-L1 therapy in patients with pretreated advanced non-small-cell lung cancer: a meta-analysis. Future Oncology. Apr 2019; 15(20): 242333.

[36] Brito AB, Camandaroba MP, de Lima VC. Anti-PD1 versus anti-PD-L1 immunotherapy in first-line therapy for advanced non-small cell lung cancer: A systematic review and meta-analysis. Thoracic Cancer. Feb 2021; 12(7): 1058-1066. 